\title{
A Fixed Point Approach to the Stability of an Integral Equation Related to the Wave Equation
}

\author{
Soon-Mo Jung \\ Mathematics Section, College of Science and Technology, Hongik University, Sejong 339-701, Republic of Korea \\ Correspondence should be addressed to Soon-Mo Jung; smjung@hongik.ac.kr
}

Received 24 July 2013; Accepted 16 October 2013

Academic Editor: Hichem Ben-El-Mechaiekh

Copyright (C) 2013 Soon-Mo Jung. This is an open access article distributed under the Creative Commons Attribution License, which permits unrestricted use, distribution, and reproduction in any medium, provided the original work is properly cited.

We will apply the fixed point method for proving the generalized Hyers-Ulam stability of the integral equation $(1 / 2 c) \int_{x-c t}^{x+c t} u\left(\tau, t_{0}\right) d \tau=u(x, t)$ which is strongly related to the wave equation.

\section{Introduction}

In 1940, Ulam [1] gave a wide ranging talk before the mathematics club of the University of Wisconsin in which he discussed a number of important unsolved problems. Among those was the question concerning the stability of group homomorphisms:

Let $G_{1}$ be a group and let $G_{2}$ be a metric group with the metric $d(\cdot, \cdot)$. Given $\varepsilon>0$, does there exist a $\delta>$ 0 such that if a function $h: G_{1} \rightarrow G_{2}$ satisfies the inequality $d(h(x y), h(x) h(y))<\delta$ for all $x, y \in G_{1}$, then there exists a homomorphism $H: G_{1} \rightarrow G_{2}$ with $d(h(x), H(x))<\varepsilon$ for all $x \in G_{1}$ ?

The case of approximately additive functions was solved by Hyers [2] under the assumption that $G_{1}$ and $G_{2}$ are the Banach spaces. Indeed, he proved that each solution of the inequality $\|f(x+y)-f(x)-f(y)\| \leq \varepsilon$, for all $x$ and $y$, can be approximated by an exact solution, say an additive function. In this case, the Cauchy additive functional equation, $f(x+$ $y)=f(x)+f(y)$, is said to have the Hyers-Ulam stability.

Rassias [3] attempted to weaken the condition for the bound of the norm of the Cauchy difference as follows:

$$
\|f(x+y)-f(x)-f(y)\| \leq \varepsilon\left(\|x\|^{p}+\|y\|^{p}\right)
$$

and proved the Hyers theorem. That is, Rassias proved the generalized Hyers-Ulam stability (or Hyers-Ulam-Rassias stability) of the Cauchy additive functional equation. (Aoki
[4] has provided a proof of a special case of Rassias' theorem just for the stability of the additive function. Aoki did not prove the stability of the linear function, which was implied by Rassias' theorem.) Since then, the stability of several functional equations has been extensively investigated [5-12].

The terminologies generalized Hyers-Ulam stability, Hyers-Ulam-Rassias stability, and Hyers-Ulam stability can also be applied to the case of other functional equations, differential equations, and various integral equations.

Let $c$ and $t_{0}$ be fixed real numbers with $c>0$. For any differentiable function $h: \mathbb{R} \times \mathbb{R} \rightarrow \mathbb{C}$, the function defined as

$$
u(x, t):=\frac{1}{2 c} \int_{x-c t}^{x+c t} h\left(\tau, t_{0}\right) d \tau
$$

is a solution of the wave equation

$$
u_{t t}(x, t)=c^{2} u_{x x}(x, t)
$$

as we see

$$
\begin{aligned}
u_{t}(x, t) & =\frac{1}{2 c} \frac{\partial}{\partial t} \int_{x-c t}^{x+c t} h\left(\tau, t_{0}\right) d \tau \\
& =\frac{1}{2} h\left(x+c t, t_{0}\right)+\frac{1}{2} h\left(x-c t, t_{0}\right), \\
u_{t t}(x, t) & =\frac{c}{2} h_{x}\left(x+c t, t_{0}\right)-\frac{c}{2} h_{x}\left(x-c t, t_{0}\right),
\end{aligned}
$$




$$
\begin{gathered}
u_{x}(x, t)=\frac{1}{2 c} h\left(x+c t, t_{0}\right)-\frac{1}{2 c} h\left(x-c t, t_{0}\right), \\
u_{x x}(x, t)=\frac{1}{2 c} h_{x}\left(x+c t, t_{0}\right)-\frac{1}{2 c} h_{x}\left(x-c t, t_{0}\right),
\end{gathered}
$$

from which we know that $u(x, t)$ satisfies the wave equation (3).

Conversely, we know that every solution $u: \mathbb{R} \times \mathbb{R} \rightarrow \mathbb{C}$ of the wave equation (3) can be expressed by

$$
u(x, t)=f(x+c t)+g(x-c t),
$$

where $f, g: \mathbb{R} \times \mathbb{R} \rightarrow \mathbb{C}$ are arbitrary twice differentiable functions. If these $f(x, t)$ and $g(x, t)$ satisfy

$$
\begin{aligned}
& \frac{1}{2 c} \int_{x-c t}^{x+c t} f(\tau) d \tau=f(x+c t), \\
& \frac{1}{2 c} \int_{x-c t}^{x+c t} g(\tau) d \tau=g(x-c t)
\end{aligned}
$$

for all $x, t \in \mathbb{R}$, then $u(x, t)$ expressed by (5) satisfies the integral equation (7). These facts imply that the integral equation (7) is strongly connected with the wave equation (3).

Cădariu and Radu [13] applied the fixed point method to the investigation of the Cauchy additive functional equation. Using such a clever idea, they could present another proof for the Hyers-Ulam stability of that equation [14-19].

In this paper, we introduce the integral equation:

$$
\frac{1}{2 c} \int_{x-c t}^{x+c t} u\left(\tau, t_{0}\right) d \tau=u(x, t),
$$

which may be considered as a special form of (2), and prove the generalized Hyers-Ulam stability of the integral equation (7) by using ideas from $[13,15,19,20]$. More precisely, assume that $\varphi(x, t)$ is a given function and $u(x, t)$ is an arbitrary and continuous function which satisfies the integral inequality:

$$
\left|\frac{1}{2 c} \int_{x-c t}^{x+c t} u\left(\tau, t_{0}\right) d \tau-u(x, t)\right| \leq \varphi(x, t) .
$$

If there exist a function $u_{0}(x, t)$ and a constant $C>0$ such that

$$
\begin{aligned}
& \frac{1}{2 c} \int_{x-c t}^{x+c t} u_{0}\left(\tau, t_{0}\right) d \tau=u_{0}(x, t), \\
& \left|u(x, t)-u_{0}(x, t)\right| \leq C \varphi(x, t),
\end{aligned}
$$

then we say that the integral equation (7) has the generalized Hyers-Ulam stability.

\section{Preliminaries}

For a nonempty set $X$, we introduce the definition of the generalized metric on $X$. A function $d: X \times X \rightarrow[0, \infty]$ is called a generalized metric on $X$ if and only if $d$ satisfies

$$
\left(M_{1}\right) d(x, y)=0 \text { if and only if } x=y \text {; }
$$

$$
\begin{aligned}
& \left(M_{2}\right) d(x, y)=d(y, x) \text { for all } x, y \in X \\
& \left(M_{3}\right) d(x, z) \leq d(x, y)+d(y, z) \text { for all } x, y, z \in X .
\end{aligned}
$$

We remark that the only one difference of the generalized metric from the usual metric is that the range of the former is permitted to include the infinity.

We now introduce one of fundamental results of fixed point theory. For the proof, we refer to [21]. This theorem will play an important role in proving our main theorems.

Theorem 1. Let $(X, d)$ be a generalized complete metric space. Assume that $\Lambda: X \rightarrow X$ is a strictly contractive operator with the Lipschitz constant $L<1$. If there exists a nonnegative integer $k$ such that $d\left(\Lambda^{k+1} x, \Lambda^{k} x\right)<\infty$ for some $x \in X$, then the following are true:

(a) the sequence $\left\{\Lambda^{n} x\right\}$ converges to a fixed point $x^{*}$ of $\Lambda$;

(b) $x^{*}$ is the unique fixed point of $\Lambda$ in

$$
X^{*}=\left\{y \in X \mid d\left(\Lambda^{k} x, y\right)<\infty\right\}
$$

(c) if $y \in X^{*}$, then

$$
d\left(y, x^{*}\right) \leq \frac{1}{1-L} d(\Lambda y, y)
$$

\section{The Generalized Hyers-Ulam Stability}

In the following theorem, for given real numbers $a, b, c$, and $t_{0}$ satisfying $c>0, t_{0}>0$, and $a+c t_{0}<b-c t_{0}$, let $I:=[a, b]$, $T:=\left(0, t_{0}\right]$, and $I_{0}:=\left[a+c t_{0}, b-c t_{0}\right]$ be finite intervals. Assume that $L$ and $M$ are positive constants with $0<L<1$. Moreover, let $\varphi: I \times T \rightarrow(0,1]$ be a continuous function satisfying

$$
\frac{1}{2 c} \int_{x-c t}^{x+c t} \varphi\left(\tau, t_{0}\right) d \tau \leq L \varphi(x, t)
$$

for all $x \in I_{0}$ and $t \in T$.

We denote by $X$ the set of all functions $f: I \times T \rightarrow \mathbb{C}$ with the following properties:

(a) $f(x, t)$ is continuous for all $x \in I_{0}$ and $t \in T$;

(b) $f(x, t)=0$ for all $x \in I \backslash I_{0}$ and $t \in T$;

(c) $|f(x, t)| \leq M \varphi(x, t)$ for all $x \in I_{0}$ and $t \in T$.

Moreover, we introduce a generalized metric on $X$ as follows:

$$
\begin{array}{r}
d(f, g):=\inf \{C \in[0, \infty]|| f(x, t)-g(x, t) \mid \\
\left.\leq C \varphi(x, t) \quad \forall x \in I_{0}, t \in T\right\} .
\end{array}
$$

Theorem 2. If a function $u \in X$ satisfies the integral inequality:

$$
\left|\frac{1}{2 c} \int_{x-c t}^{x+c t} u\left(\tau, t_{0}\right) d \tau-u(x, t)\right| \leq \varphi(x, t)
$$


for all $x \in I_{0}$ and $t \in T$, then there exists a unique function $u_{0} \in X$ which satisfies

$$
\begin{gathered}
\frac{1}{2 c} \int_{x-c t}^{x+c t} u_{0}\left(\tau, t_{0}\right) d \tau=u_{0}(x, t), \\
\left|u(x, t)-u_{0}(x, t)\right| \leq \frac{1}{1-L} \varphi(x, t)
\end{gathered}
$$

for all $x \in I_{0}$ and $t \in T$.

Proof. First, we show that $(X, d)$ is complete. Let $\left\{h_{n}\right\}$ be a Cauchy sequence in $(X, d)$. Then, for any $\varepsilon>0$ there exists an integer $N_{\varepsilon}>0$ such that $d\left(h_{m}, h_{n}\right) \leq \varepsilon$ for all $m, n \geq N_{\varepsilon}$. In view of (13), we have

$$
\begin{gathered}
\forall \varepsilon>0, \quad \exists N_{\varepsilon} \in \mathbb{N}, \forall m, n \geq N_{\varepsilon}, \forall x \in I_{0}, \forall t \in T: \\
\left|h_{m}(x, t)-h_{n}(x, t)\right| \leq \varepsilon \varphi(x, t) .
\end{gathered}
$$

If $x$ and $t$ are fixed, (17) implies that $\left\{h_{n}(x, t)\right\}$ is a Cauchy sequence in $\mathbb{C}$. Since $\mathbb{C}$ is complete, $\left\{h_{n}(x, t)\right\}$ converges for any $x \in I_{0}$ and $t \in T$. Thus, considering (b), we can define a function $h: I \times T \rightarrow \mathbb{C}$ by

$$
h(x, t):=\lim _{n \rightarrow \infty} h_{n}(x, t), \quad(x \in I, t \in T) .
$$

Since $\varphi$ is bounded on $I_{0} \times T$, (17) implies that $\left\{\left.h_{n}\right|_{I_{0} \times T}\right\}$ converges uniformly to $\left.h\right|_{I_{0} \times T}$ in the usual topology of $\mathbb{C}$. Hence, $h$ is continuous and $|h|$ is bounded on $I_{0} \times T$ with an upper bound $M \varphi(x, t)$; that is, $h \in X$. (It has not been proved yet that $\left\{h_{n}\right\}$ converges to $h$ in $(X, d)$.)

If we let $m$ increase to infinity, it follows from (17) that

$$
\begin{gathered}
\forall \varepsilon>0, \quad \exists N_{\varepsilon} \in \mathbb{N}, \forall n \geq N_{\varepsilon}, \forall x \in I_{0}, \quad \forall t \in T: \\
\left|h(x, t)-h_{n}(x, t)\right| \leq \varepsilon \varphi(x, t) .
\end{gathered}
$$

By considering (13), we get

$$
\forall \varepsilon>0, \exists N_{\varepsilon} \in \mathbb{N}, \forall n \geq N_{\varepsilon}: d\left(h, h_{n}\right) \leq \varepsilon .
$$

This implies that the Cauchy sequence $\left\{h_{n}\right\}$ converges to $h$ in $(X, d)$. Hence, $(X, d)$ is complete.

We now define an operator $\Lambda: X \rightarrow X$ by

$$
(\Lambda h)(x, t):= \begin{cases}\frac{1}{2 c} \int_{x-c t}^{x+c t} h\left(\tau, t_{0}\right) d \tau & \left(x \in I_{0}, t \in T\right), \\ 0 & \text { (otherwise) }\end{cases}
$$

for all $h \in X$. Then, according to the fundamental theorem of calculus, $\Lambda h$ is continuous on $I_{0} \times T$. Furthermore, it follows from (12), (c), and (21) that

$$
\begin{aligned}
|(\Lambda h)(x, t)| & \leq \frac{1}{2 c} \int_{x-c t}^{x+c t}\left|h\left(\tau, t_{0}\right)\right| d \tau \\
& \leq \frac{1}{2 c} \int_{x-c t}^{x+c t} M \varphi\left(\tau, t_{0}\right) d \tau \\
& \leq M L \varphi(x, t)<M \varphi(x, t)
\end{aligned}
$$

for any $x \in I_{0}$ and $t \in T$. Hence, we conclude that $\Lambda h \in X$.
We assert that $\Lambda$ is strictly contractive on $X$. Given any $f, g \in X$, let $C_{f g} \in[0, \infty]$ be an arbitrary constant with $d(f, g) \leq C_{f g}$. That is,

$$
|f(x, t)-g(x, t)| \leq C_{f g} \varphi(x, t)
$$

for all $x \in I_{0}$ and $t \in T$. Then, it follows from (12), (21), and (23) that

$$
\begin{aligned}
\mid(\Lambda f) & (x, t)-(\Lambda g)(x, t) \mid \\
\quad & \frac{1}{2 c}\left|\int_{x-c t}^{x+c t}\left(f\left(\tau, t_{0}\right)-g\left(\tau, t_{0}\right)\right) d \tau\right| \\
& \leq \frac{1}{2 c} \int_{x-c t}^{x+c t}\left|f\left(\tau, t_{0}\right)-g\left(\tau, t_{0}\right)\right| d \tau \\
& \leq \frac{C_{f g}}{2 c} \int_{x-c t}^{x+c t} \varphi\left(\tau, t_{0}\right) d \tau \\
& \leq L C_{f g} \varphi(x, t)
\end{aligned}
$$

for all $x \in I_{0}$ and $t \in T$. That is, $d(\Lambda f, \Lambda g) \leq L C_{f g}$. Hence, we may conclude that $d(\Lambda f, \Lambda g) \leq L d(f, g)$ for any $f, g \in X$ and we note that $0<L<1$.

We prove that the distance between the first two successive approximations of $\Lambda$ is finite. Let $h_{0} \in X$ be given. By (b), (c), and (13) and from the fact that $\Lambda h_{0} \in X$, we have

$$
\begin{aligned}
\left|\left(\Lambda h_{0}\right)(x, t)-h_{0}(x, t)\right| & \leq\left|\left(\Lambda h_{0}\right)(x, t)\right|+\left|h_{0}(x, t)\right| \\
& \leq 2 M \varphi(x, t)
\end{aligned}
$$

for any $x \in I_{0}$ and $t \in T$. Thus, (13) implies that

$$
d\left(\Lambda h_{0}, h_{0}\right) \leq 2 M<\infty .
$$

Therefore, it follows from Theorem 1(a) that there exists a $u_{0} \in X$ such that $\Lambda^{n} h_{0} \rightarrow u_{0}$ in $(X, d)$ and $\Lambda u_{0}=u_{0}$.

In view of (c) and (13), it is obvious that $\{f \in X$ । $\left.d\left(h_{0}, f\right)<\infty\right\}=X$, where $h_{0}$ was chosen with the property (26). Now, Theorem $1(\mathrm{~b})$ implies that $u_{0}$ is the unique element of $X$ which satisfies $\left(\Lambda u_{0}\right)(x, t)=u_{0}(x, t)$ for any $x \in I_{0}$ and $t \in T$. that

Finally, Theorem 1(c), together with (13) and (14), implies

$$
d\left(u, u_{0}\right) \leq \frac{1}{1-L} d(\Lambda u, u) \leq \frac{1}{1-L}
$$

since (14) means that $d(\Lambda u, u) \leq 1$. In view of (13), we can conclude that (16) holds for all $x \in I_{0}$ and $t \in T$.

Remark 3. Even though condition (12) seems to be strict, the condition can be satisfied provided that $a$ and $b$ are chosen so that $|b-a|$ is small enough and $c$ is a large number.

\section{Conflict of Interests}

The author declares that there is no conflict of interests regarding the publication of this paper. 


\section{Acknowledgments}

The author would like to express his cordial thanks to the referees for useful remarks. This research was supported by Basic Science Research Program through the National Research Foundation of Korea (NRF) funded by the Ministry of Education (no. 2013R1A1A2005557).

\section{References}

[1] S. M. Ulam, A Collection of Mathematical Problems, Interscience Publishers, New York, NY, USA, 1960.

[2] D. H. Hyers, "On the stability of the linear functional equation," Proceedings of the National Academy of Sciences of the United States of America, vol. 27, no. 4, pp. 222-224, 1941.

[3] T. M. Rassias, "On the stability of the linear mapping in Banach spaces," Proceedings of the American Mathematical Society, vol. 72, pp. 297-300, 1978.

[4] T. Aoki, "On the stability of the linear transformation in Banach spaces," Journal of the Mathematical Society of Japan, vol. 2, no. 1-2, pp. 64-66, 1950.

[5] L. Cădariu, “The generalized Hyers-Ulam stability for a class of the Volterra nonlinear integral equations," Buletinul Stiintific al Universitatii "Politehnica" din Timisoara, vol. 56, no. 70, pp. 3038, 2011.

[6] L. Cǎdariu, L. Gǎvruţa, and P. Gǎvruţa, "Weighted space method for the stability of some nonlinear equations," Applicable Analysis and Discrete Mathematics, vol. 6, no. 1, pp. 126-139, 2012.

[7] G. L. Forti, "Hyers-Ulam stability of functional equations in several variables," Aequationes Mathematicae, vol. 50, no. 1-2, pp. 143-190, 1995.

[8] P. Găvrută, "A generalization of the Hyers-Ulam-Rassias stability of approximately additive mappings," Journal of Mathematical Analysis and Applications, vol. 184, no. 3, pp. 431-436, 1994.

[9] D. H. Hyers, G. Isac, and M. Th. Rassias, Stability of Functional Equations of Several Variables, Birkhäuser, Basel, Switzerland, 1998.

[10] D. H. Hyers and T. M. Rassias, "Approximate homomorphisms," Aequationes Mathematicae, vol. 44, no. 2-3, pp. 125-153, 1992.

[11] S.-M. Jung, Hyers-Ulam-Rassias Stability of Functional Equations in Nonlinear Analysis, vol. 48 of Springer Optimization and Its Applications, Springer, New York, NY, USA, 2011.

[12] T. M. Rassias, "On the stability of functional equations and a problem of Ulam," Acta Applicandae Mathematicae, vol. 62, no. 1, pp. 23-130, 2000.

[13] L. Cădariu and V. Radu, "On the stability of the Cauchy functional equation: a fixed point approach," Grazer Mathematische Berichte, vol. 346, pp. 43-52, 2004.

[14] M. Akkouchi, "Hyers-Ulam-Rassias stability of nonlinear Volterra integral equations via a fixed point approach," Acta Universitatis Apulensis, vol. 26, pp. 257-266, 2011.

[15] L. Cădariu and V. Radu, "Fixed points and the stability of Jensen's functional equation," Journal of Inequalities in Pure and Applied Mathematics, vol. 4, no. 1, Article ID 4, 2003.

[16] L. P. Castro and A. Ramos, "Hyers-Ulam-Rassias stability for a class of nonlinear Volterra integral equations," Banach Journal of Mathematical Analysis, vol. 3, no. 1, pp. 36-43, 2009.

[17] K. Ciepliński, "Applications of fixed point theorems to the Hyers-Ulam stability of functional equations-a survey," Annals of Functional Analysis, vol. 3, no. 1, pp. 151-164, 2012.
[18] S.-M. Jung, "A fixed point approach to the stability of isometries," Journal of Mathematical Analysis and Applications, vol. 329, no. 2, pp. 879-890, 2007.

[19] V. Radu, "The fixed point alternative and the stability of functional equations," Fixed Point Theory, vol. 4, no. 1, pp. 9196, 2003.

[20] S.-M. Jung, "A fixed point approach to the stability of a volterra integral equation," Fixed Point Theory and Applications, vol. 2007, Article ID 57064, 9 pages, 2007.

[21] B. Margolis and J. Diaz, "A fixed point theorem of the alternative for contractions on a generalized complete metric space," Bulletin of the American Mathematical Society, vol. 74, pp. 305309, 1968. 


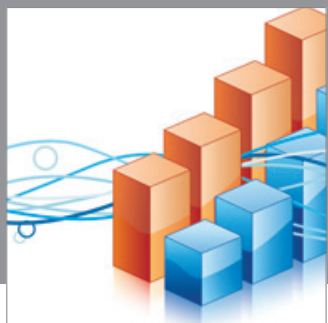

Advances in

Operations Research

mansans

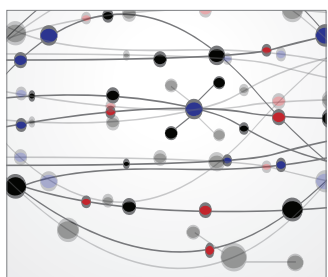

The Scientific World Journal
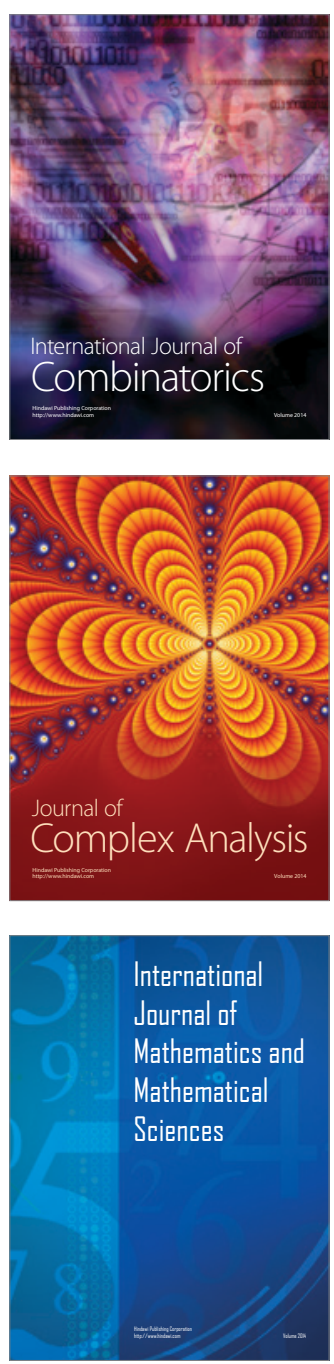
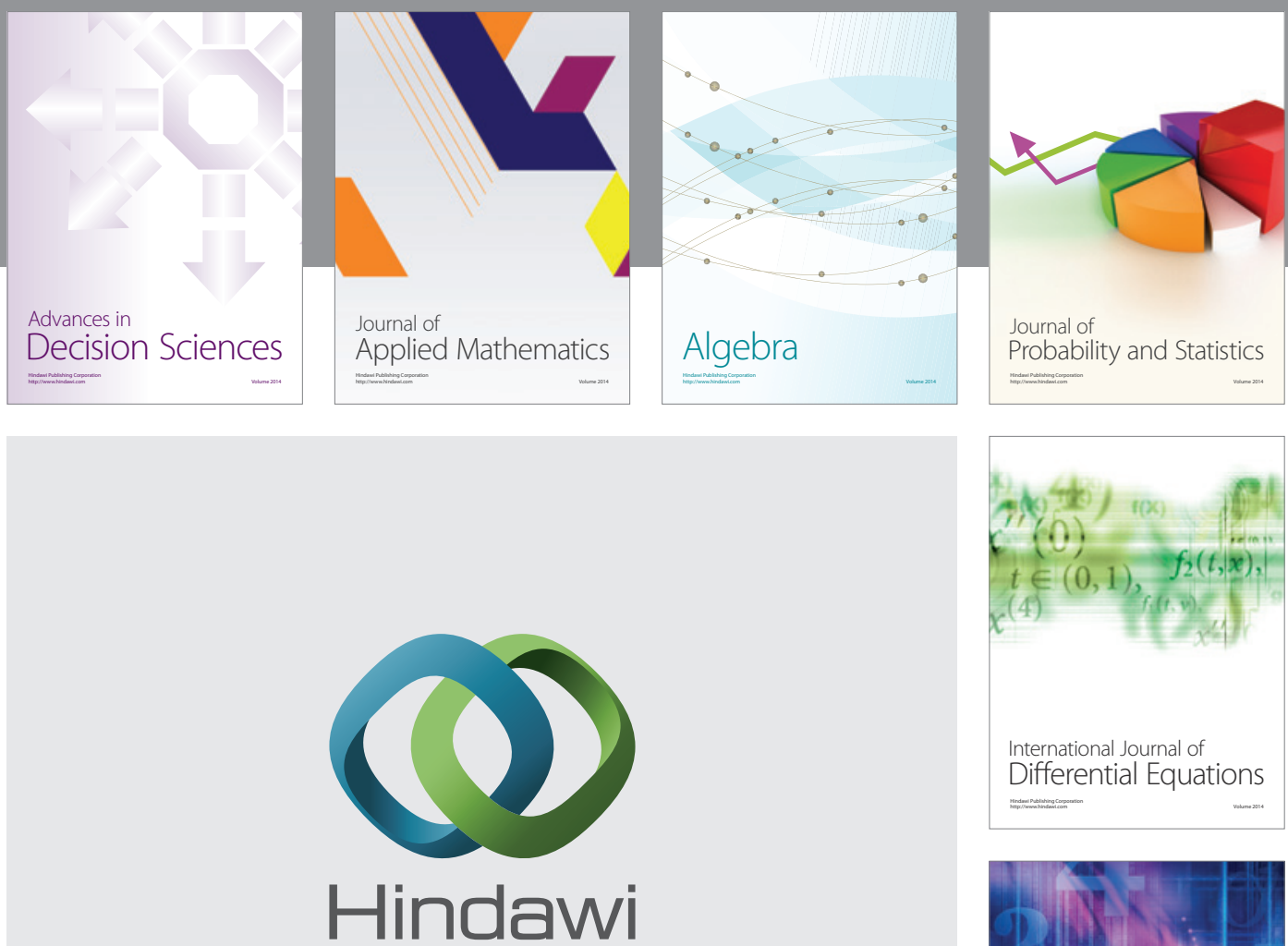

Submit your manuscripts at http://www.hindawi.com
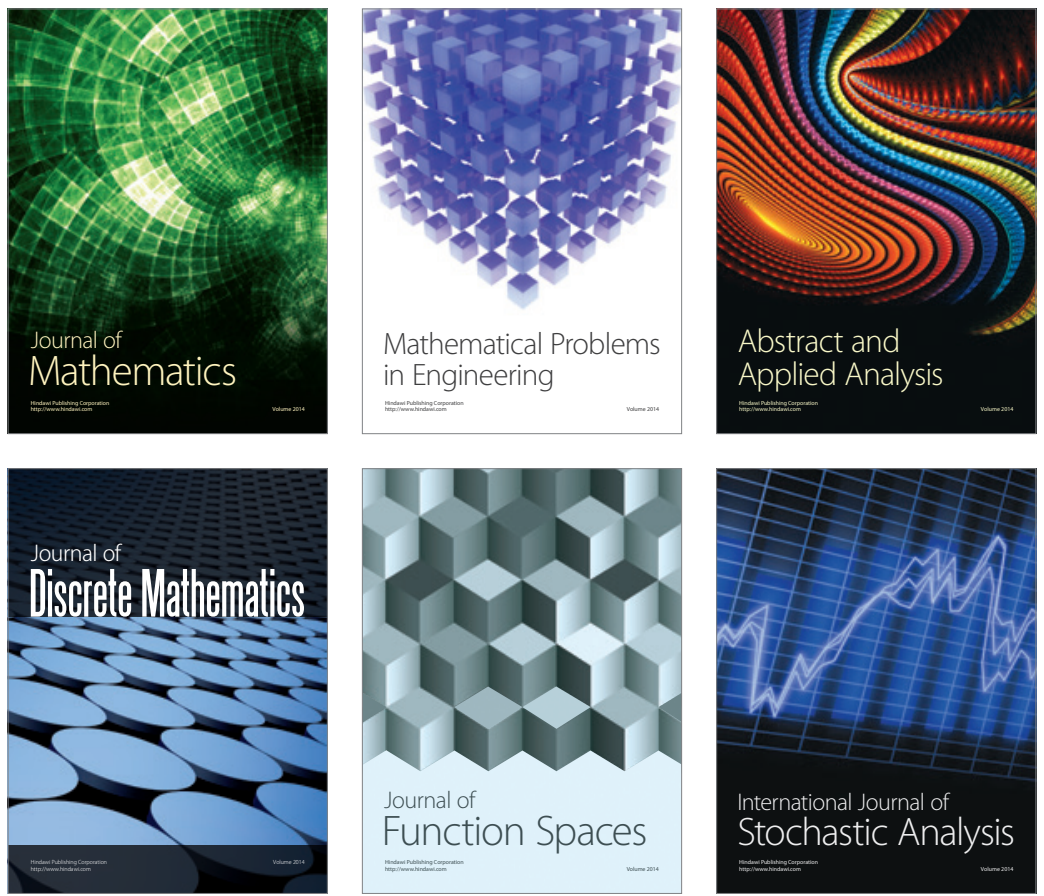

Journal of

Function Spaces

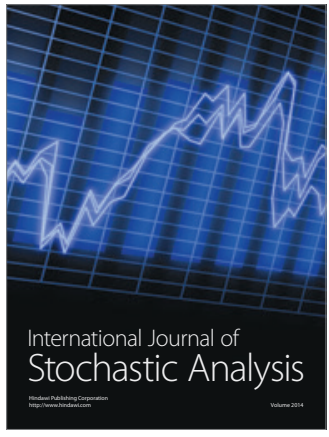

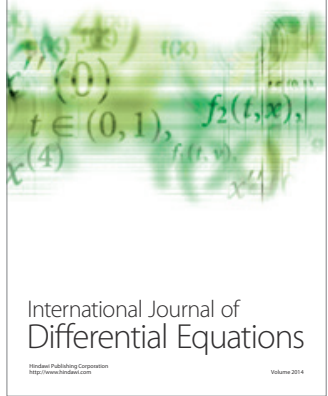
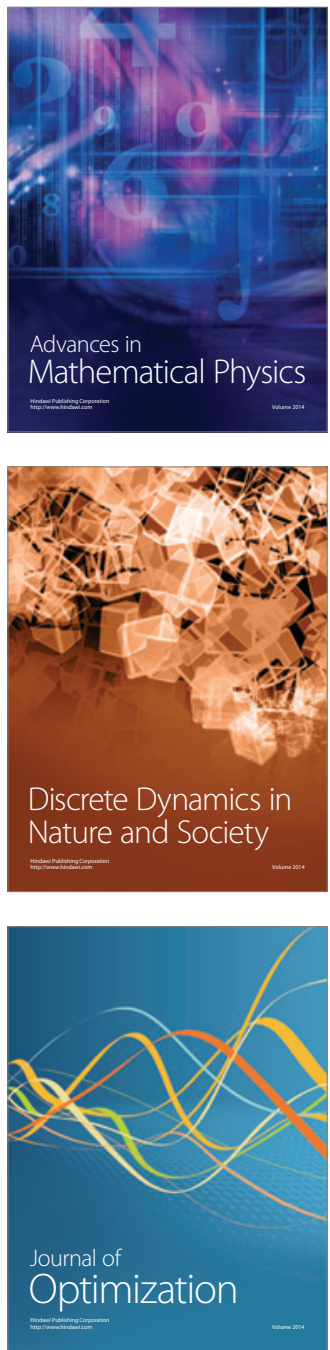